\title{
Enquête
}

Archives de la revue Enquête

6 | 1991

La socialisation de la jeunesse

\section{Jeunes Maghrébins}

Un groupe sociologiquement pertinent?

Jean-Pierre Zirotti

\section{(2) OpenEdition}

Journals

Édition électronique

URL : http://journals.openedition.org/enquete/166

DOI : 10.4000/enquete. 166

ISSN : 1953-809X

Éditeur :

Cercom, Éditions Parenthèses

Édition imprimée

Date de publication : 2 juin 1991

Référence électronique

Jean-Pierre Zirotti, « Jeunes Maghrébins », Enquête [En ligne], 6 | 1991, mis en ligne le 27 juin 2013, consulté le 21 avril 2019. URL : http://journals.openedition.org/enquete/166 ; DOI : 10.4000/ enquete. 166

Ce document a été généré automatiquement le 21 avril 2019 


\title{
Jeunes Maghrébins
}

\author{
Un groupe sociologiquement pertinent?
}

\author{
Jean-Pierre Zirotti
}

1 À l'occasion d'un colloque dans le cadre duquel R. Establet avait bien voulu jouer le rôle de discutant d'un texte introductif au débat sur la question de la scolarisation des « immigrés » (Zirotti, 1988), celui-ci remit en question la pertinence sociologique de la catégorie "enfants de travailleurs immigrés» (Establet, 1988). Il n'était pas alors explicitement question de "Maghrébins " mais d'«enfants de travailleurs immigrés ", dont ils représentaient d'ailleurs l'essentiel des effectifs enquêtés. Establet soulignait l'effet de stigmatisation que pourrait produire une analyse qui tendrait à spécifier négativement ces élèves et craignait que la comparaison des performances et des cheminements scolaires des enfants de travailleurs français et immigrés ne conduise à masquer des différences plus fortes et plus significatives. Il rappelait la nature de la culture scolaire et sa place dans une logique de " reproduction ", et par là situait ces deux publics scolaires dans une même distance de classe à l'univers scolaire.

2 Or le texte soumis à discussion s'inspirait de recherches qui avaient dégagé, notamment par le recours à l'analyse de dépendance causale, l'existence d'un processus d'orientation scolaire discriminatoire entre "enfants de travailleurs français» et "enfants de travailleurs immigrés", à la défaveur de ces derniers (Zirotti \& Novi, 1979). L'objectivation statistique, dans le cadre d'une démarche comparative, instituait dans un premier niveau de pertinence les deux catégories d'élèves opposées. Il était attesté qu'au plan $\mathrm{du}$ traitement scolaire l'origine nationale était un facteur pertinent de différenciation au sein d'une population homogène, autant que faire se pouvait, dans son origine ouvrière.

3 La question n'était donc plus de savoir si l'opposition entre l'origine nationale et l'origine étrangère était recevable mais de comprendre comment certaines caractéristiques sociales des élèves « immigrés », après les avoir identifiées, pouvaient être porteuses d'un tel effet.

4 Mais dans tous ces travaux c'est, au principal, d'élèves issus de l'immigration maghrébine qu'il était question, tant parce qu'ils étaient très majoritairement constitutifs des populations «immigrées » prises en considération que parce que leur forte «visibilité 
sociale » s'accordait à une position originale, remarquée et soulignée, au sein de l'école. Bien sûr, la "visibilité » n'est pas un critère sociologique mais une question ouverte à l'investigation. Traiter cette question c'est répondre à Establet et soutenir, en accord partiel avec lui, que ce n'est pas tant la distance à la culture scolaire qui fait la différence entre ces deux populations, que le statut scolaire de certains "immigrés», les «Maghrébins » notamment. En effet le processus discriminatoire que nous avons établi s'alimente, avant de les renforcer en retour, des représentations dévalorisantes importées de l'extérieur de l'univers scolaire. La stigmatisation particulière des "Maghrébins " trouve son origine dans l'histoire de ce courant migratoire et dans son traitement sociopolitique, mais c'est par réinterprétation de ces attributions catégorielles dans une logique propre à l'univers scolaire qu'est constituée leur spécificité. De la déprivation culturelle à l'obscurantisme et au fanatisme religieux (Zirotti, 1990), des formes changeantes d'une essence maghrébine supposée ont tour à tour fourni en arguments la stigmatisation de ces élèves et armé des pratiques discriminatoires au sein même de l'école de la République. Ils menaceraient le niveau et la quiétude des établissements scolaires comme les «bandes tribales » chères au Front national menaceraient la qualité de vie des banlieues et la sérénité des centres commerciaux.

5 Aujourd'hui, dans les media, le thème de la « violence dans les banlieues » a pris le relais de l'« affaire du foulard islamique ». Et sont à nouveau convoqués les spécialistes du social pour dire ce qu'il en est des causes et des effets, avec une interrogation récurrente, plus ou moins manifeste, sur la part de l'immigration dans de tels événements, et pour se frotter, sur ce point notamment, aux discours politiques les plus extrémistes. À cette occasion plusieurs sociologues ont présenté dans la presse des points de vue professionnels, constitués sur la base d'études de la jeunesse, des formes contemporaines de la marginalité sociale, etc. Organisé autour de l'acceptation ou du rejet d'un naturalisme social qui ferait de certaines catégories d'immigrés et, plus particulièrement de leurs descendants des fauteurs de troubles potentiels, ce débat tend à s'enfermer dans une oscillation entre deux positions schématiques. Ce balancement, caricaturé peut-être dans l'exercice journalistique, n'est pourtant pas sans rappeler les difficultés auxquelles se heurte l'analyse sociologique dès qu'elle inclut dans sa population des groupes sociaux issus de l'immigration.

6 On peut se demander si la difficulté n'est pas plus grande lorsque les phénomènes sur lesquels porte le regard du sociologue ont le statut d'expression, dans le cadre urbain, des difficultés de l'insertion sociale et professionnelle de certaines composantes des jeunes générations. Si l'approche objectivante a constitué une base pertinente de l'interrogation sur la spécificité du statut scolaire de certains groupes d'élèves, elle ne semble plus pouvoir introduire aux mêmes interrogations dès lors que les jeunes des quartiers populaires, indépendamment de leurs origines nationales, paraissent confrontés à des mêmes formes d'exclusion sociale.

7 Sauf à interroger la qualité des descriptions statistiques qui, saisissant dans leur aspect le plus «objectif» les trajectoires scolaires, professionnelles ou résidentielles, font l'économie de l'analyse micro-sociologique des expériences qui en ont constitué les étapes, on est conduit à subsumer l'identité des causes dans celle des effets observables.

8 Le risque est grand de voir une opposition fondée à certaines dérives du discours politique, qui lui s'alimente des catégories du sens commun pour dénoncer sur le critère d'« évidentes » différences de "physionomie » les «gangs immigrés » et les "bandes tribales $»^{1}$, nier l'efficacité propre de caractéristiques sociales telles que l'origine 
nationale, culturelle ou l'affiliation religieuse. À tant nier toute spécificité culturelle et à négliger la dynamique complexe des appartenances et des affiliations, outre le risque d'une erreur d'analyse, ne propose-t-on pas une interprétation simpliste de la réalité sociale et ne renforce-t-on pas la conviction raciste en accréditant l'illégitimité de toute manifestation d'une quelconque spécificité comme l'affaire $d u$ « foulard islamique » en a été le révélateur? En effet, dès lors qu'est proclamée, par exemple, l'« assimilation » des jeunes «Maghrébins» (Lapeyronnie, 1987), toute affirmation identitaire ou religieuse peut être frappée du sceau de l'archaïsme si ce n'est de celui du fanatisme, à tout le moins perçue comme une résurgence anormale.

9 La neutralisation de la variable «ethnique » dans la commune condition des jeunes des grands ensembles populaires, qui n'est pas unanimement attestée (Blöss, 1989), ne saurait pour autant permettre d'exclure de l'investigation sociologique ce qui a pour le moins statut de trait social pertinent dans les classements sociaux de sens commun. Être «Black» ou «Beur », «Antillais » ou « Maghrébin » est une caractéristique constitutive de l'expérience sociale de ces jeunes, autant comme critère de classement subi que comme ressource interprétative de leurs expériences ou horizon d'une affirmation identitaire. Mais ce ne sont pas des caractéristiques permutables, même si elles peuvent participer d'un même processus de stigmatisation; dotées chacunes d'une profondeur historique particulière et riches de ressources symboliques originales, il ne convient pas de les confondre, aussi mon analyse ne porte-t-elle que sur l'usage de la seule catégorie « jeunes Maghrébins ».

10 Que signifie l'expression « jeunes Maghrébins »? Il est évident que les termes constitutifs de cette locution sont imprécis. D'autres ont déjà abondamment questionné la notion de «jeunes $»^{2}$; on peut proposer de voir redoubler ici son usage habituel, comme un âge de la vie aux limites imprécises, par l'indication d'une origine. Mais ceci n'est approprié et compréhensible que par référence à un contexte social marqué par la présence de groupes sociaux immigrés; dans le cas français, aujourd'hui, "jeunes Maghrébins » désigne les fils et les filles de Maghrébins, donc la descendance d'un courant migratoire.

11 Le "Maghrib» est à l'origine une catégorie de la géographie arabe; le géographe Muqaddasî, au $\mathrm{x}^{\mathrm{e}}$ siècle, désigne par ce terme les territoires du monde musulman qui, à l'époque abbaside, s'étendent de l'Andalousie à l'Égypte (Miquel, 1977). Sous l'effet de l'emprise coloniale française sur les territoires qui donneront naissance à l'Algérie, au Maroc et à la Tunisie, la catégorie géographique a été réduite pour faire des «Maghrébins» les immigrés originaires de ces trois pays. C'est bien là un exemple typique de la capacité d'imposition du dominant qui désigne l'origine et la réduit à une catégorie fonctionnelle pour lui. Dès lors qu'il ne gère plus des populations « indigènes " mais des "immigrés », un espace géographique vaste est une référence suffisante pour situer une origine sans s'encombrer de distinctions nationales et encore moins culturelles ou ethniques. Si l'administration coloniale a favorisé des classements plus subtils et a su, par exemple, distinguer les Kabyles des Arabes, dans le temps de l'immigration postcoloniale, cette catégorie globale suffit aux distinctions sociales les plus courantes et joue comme un équivalent à « musulmans ».

12 Mais l'usage "éclairé » des qualificatifs disponibles peut s'affranchir des charges sémantiques socio-historiquement constituées pour ne reprendre du stigmate, par exemple, que la délimitation du groupe social stigmatisé et en faire, de ce point de vue, une catégorie pertinente de l'analyse sociologique. Si l'axe paradigmatique constitué des locutions mobilisées pour désigner ces populations connaît quelques variantes selon que 
l'identification privilégie le statut socio-culturel, l'appartenance religieuse, l'origine nationale ou l'origine culturelle, toutes ont en commun d'imposer des regroupements sous des «labels » qui peuvent prêter à stigmatisation. Ce sont bien sûr les conditions sociales de l'usage de ces locutions qui en déterminent l'orientation et de ce point de vue la première spécificité de ces "jeunes Maghrébins" est d'être, par cette origine-là, stigmatisables. Si le sociologue prend à son compte cette catégorie, ce n'est pas dans son utilisation ordinaire et éventuellement stigmatisante mais pour cette première raison que, servant à de telles fins, elle construit la population qu'elle désigne. Le retournement du stigmate qui, par une inversion des syllabes propre au verlan, s'exprime au travers d'une nouvelle «étiquette»- «Beurs»-, est lu comme une forme d'unification symbolique de ces «jeunes Maghrébins », descendants d'immigrés, opérée sur la base du rejet et de l'exclusion (Streiff-Fénart, 1987) ${ }^{3}$.

La question à laquelle nous sommes confrontés n'est pas étrangère à celle du rapport entre catégorisation professionnelle et classements sociaux (Desrosières \& Thévenot, 1988) : toutes deux portent sur la nature de la relation entre connaissance savante et connaissance ordinaire. L'approche cognitive comme l'interactionnisme et l'ethnométhodologie ont largement mis en question les descriptions savantes du social opérées sans prendre en compte l'activité « constructive» des sujets sociaux au travers de leurs représentations, de leurs jugements et de leurs raisonnements. Mais lorsque Conein s'interroge sur l'unité des domaines de la catégorisation sociale, à partir d'un point de vue cognitiviste qui propose que les conceptualisations liées aux classements sociaux résulteraient d'une capacité identique à conceptualiser (Turiel, 1983), en opposant les groupes sociaux selon qu'ils relèvent ou non d'une « identification à ancrage perceptuel ", ne surévalue-t-il pas le caractère d'évidence des appartenances générationnelles, parentales, ethniques par rapport aux appartenances professionnelles (Conein, 1990) ? Si le principe organisateur des catégories de nature conventionnelle, comme les professions, est de type critériel ou nominal (Keil, 1989 cité par Conein), la perception visuelle de caractères physiques constitutifs du sexe, de l'âge ou de la « race » n'est pas indépendante d'un accomplissement social et interactif de ceux-ci (Garfinkel, 1967).

Le poids de la constitution sociale est d'autant plus fort que, pour ce qui nous concerne ici, il n'est pas question de "race", même si l'on entend par là désigner l'efficacité classificatoire d'un caractère physique particulièrement évident comme la couleur de la peau, mais d'une origine nationale et culturelle non accompagnée de traits physiques constitutifs d'un type humain physiquement spécifique, au plus associée à une certaine fréquence de réalisation de traits physiques "méditerranéens " que la discrimination sociale tend à ériger en critère évident d'une nature humaine spécifique ${ }^{4}$.

Dans les travaux portant sur les jeunes des milieux populaires partageant une communauté de résidence dans les «banlieues ", on peut trouver l'exemple d'analyses qui se sont à des degrés divers préoccupées de privilégier le point de vue de ces acteurs (Lagrée \& Lew-Fai, 1985; Dubet, 1987). J.-C. Lagrée et P. Lew-Fai, au terme d'une recherche sur une population de jeunes de 15 à 20 ans frappés par l'exclusion scolaire et professionnelle, concluent que leur mode privilégié de rapport au social est structuré par l'appartenance à des «bandes ». Celles-ci sont le plus couramment «multi-ethniques », à l'exception de la bande des "Blacks» composée de jeunes Antillais, Réunionnais et Guadeloupéens, et de la bande des "Rabeux» exclusivement maghrébine dans sa composition masculine. 


\section{La clôture oppositionnelle de la bande des « Blacks »}

«s'effectue au nom des capacités, de la culture, de la mentalité des Noirs réunionnais, antillais [...] [C']est le moyen d'affirmer une identité culturelle et de se positionner par ces références culturelles » (Lagrée \& Lew-Fai, 1985, p. 110).

Selon les auteurs, si les «Blacks» ont l'initiative d'une catégorisation qui s'impose aux autres membres de leur espace résidentiel, il n'en est pas de même pour les « Rabeux » :

«L'enfermement des jeunes dans leur ethnicité n'est donc pas, ici, la marque d'une revendication ou d'un positionnement culturel. Il est le résultat des rapports d'exclusion et de démarquage qui structurent l'univers des jeunes. Il est le corrélat de la place qu'ils occupent dans les rapports sociaux. » (Ibid., p. 111.)

Deux formes de rapport à la ressource "ethnique", après avoir reçu un statut d'exception, sont constituées. Si la première s'alimente d'une consistance culturelle, la seconde n'est que réactive à la stigmatisation. On peut s'étonner que les «Blacks » ne soient pas soumis à un même marquage social que les Maghrébins et peut-être eût-il fallu, au moins, s'interroger sur d'éventuelles variations contextuelles et culturelles propres à l'engagement de chacun de ces groupes dans l'activité langagière suscitée par l'enquête.

19 F. Dubet au terme d'une intervention sociologique qui associa des jeunes, immigrés ou non, en diverses banlieues, rend compte de cette forme déstructurée de rapport au social que serait la "galère ». Apparemment absents parce qu'inclus dans la catégorie générale « jeunes immigrés », les « jeunes Maghrébins » se découvrent, dans le détail des analyses, comme les acteurs principaux de ce groupe social. Mais cette indifférenciation qui brouille les origines et les éventuelles différences de statut social ne s'oppose pas à l'inscription d'un certain particularisme de cette population au sein même du processus de la « galère » :

«La spécificité des jeunes immigrés, d'origine maghrébine pour la plupart ${ }^{5}$, dégagée par cette recherche est une double accentuation des logiques de la galère [...] Il semble que l'immigration accentue simultanément les processus de désorganisation sociale et les possibilités de créer des espaces de résistance [...] Ils apparaissent comme doublement centraux dans les formes d'action sociale des jeunes des milieux populaires [...] ils sont souvent les éléments les plus dynamiques des groupes, à la fois dans la marginalité et dans les tentatives d'action collective.» (Dubet, 1987, p. 327.)

Mais de quelle spécificité est-il question? F. Dubet récuse le parti pris «culturaliste » de la spécificité culturelle et veut se dégager des descriptions « misérabilistes » qui auraient longtemps organisé les points de vue sur l'immigration. L'accumulation des inégalités serait partagée avec tous les jeunes de ces mêmes quartiers et

«... la spécificité des jeunes immigrés par rapport à leurs camarades français n'est pas tant l'exclusion que ses fondements ethniques [...] Les jeunes immigrés constituent une population cible où l'accumulation des inégalités est d'autant plus vivement ressentie qu'elle est associée au racisme quotidien, au déni de justice et, souvent, à la violence ouverte. » (Ibid., p. 331.)

21 L'altérité est donc bien constituée dans cette analyse, au terme d'une intervention sociologique qui a associé dans des groupes de réflexion des membres de la population enquêtée et des sociologues. C'est de ce travail en commun que l'analyste dégage son savoir. Sans revenir sur les caractéristiques de cette conception de l'analyse sociologique initiée par A.Touraine, on peut tenter d'en apprécier les conséquences sur la connaissance produite. La spécificité ainsi constituée est dégagée des descriptions produites par les acteurs, mais un travail qui prend pour objet l'activité langagière des sujets ne peut se satisfaire de ponctuer le compte rendu qui en est fait de locutions 
- «d'après ce qu'ils disent », «selon ces jeunes »- qui tendent à garantir la fidélité de l'interprétation. En n'exposant pas le travail qui de la parole de ces jeunes a constitué celle du sociologue, on interdit toute connaissance de leurs discours comme de ce qui était en jeu dans les conditions de leur expression. Ce faisant, une orientation de recherche bien intentionnée s'expose à la critique d'être «en continuité avec la connaissance de sens commun, puisqu'elle n'est qu'une “construction des constructions" » (Bourdieu, 1987, p. 149).

Guidé par la volonté théorique de rompre avec le substantialisme qui a marqué nombre d'approches sociologiques de l'immigration, F. Dubet dépouille, a priori, de toute consistance la catégorie sociale qui va s'imposer à lui au cours de son intervention. Ces jeunes Maghrébins, puisqu'il s'agit principalement d'eux, ne sont reconnus qu'à titre d'acteurs exemplaires de la "galère ", plus en rage parce que confrontés à des formes plus brutales d'exclusion que les Français, plus actifs parce qu'ils

«se perçoivent comme la seule force d'action autonome capable de parler au nom de l'immigration. Les plus enragés sont aussi les plus militants [...] La spécificité des jeunes immigrés apparaît moins centrée sur la culture que sur l'accentuation de certaines contraintes sociales » (Dubet, 1987, p. 333).

Leur spécificité est donc mesurée à l'aune de la stigmatisation et de l'exclusion dont ils sont l'objet; ils ne seraient constitués que par la forme « raciste » d'une exclusion sociale, par ailleurs partagée avec d'autres. Et les mouvements sociaux auxquels ils ont donné naissance (Marche pour l'égalité des droits en 1983, Convergence pour une France multiraciale et multiculturelle en 1984) seraient dépourvus de toutes autres ressources mobilisatrices.

C'est là un mode d'existence singulier réduit à une "visibilité sociale " construite en réaction à une discrimination qui serait d'autant plus forte que l'assimilation de ces jeunes serait grande (Lapeyronnie, 1987). Or la discrimination explicite et brutale ne peut susciter le repliement "communautaire", l'affirmation identitaire, la revendication « ethnique » ou religieuse, que si des ressources instituées sans lesquelles il n'est ni repli, ni affirmation, ni revendication, sont disponibles (Oriol, 1989). C'est dire que la spécificité n'est pas que réactive, elle s'alimente d'une altérité mobilisable qui peut trouver ses ressources tant dans une origine nationale ou culturelle, que dans une affiliation ou solidarité religieuse.

Si le premier degré de pertinence sociologique de la catégorie «jeunes Maghrébins » est constitué par le traitement social dont ils sont l'objet, le second degré de pertinence relève de l'existence d'un univers spécifique de ressources symboliques (des références nationales, culturelles et religieuses), mobilisables, au gré des circonstances, tant pour l'attribution que pour la revendication d'une altérité.

\section{BIBLIOGRAPHIE}

Blöss T. - « Jeunes Maghrébins des quartiers nord de Marseille. Une génération charnière », in Familles, générations, patrimoines, $\mathrm{n}^{\circ} \mathrm{sp}$. de : Les Annales de la Recherche urbaine, 41, 1989, p. 59-66. 
BlöSS T. \& FERONI I. - « Jeunesse : objet politique, objet biographique », Enquête. Cahiers du CERCOM, 6, 1991, p. 145-184.

BOURDIEU P. - Choses dites, Paris, Minuit, 1987.

CONEIN B. - « Catégorisation professionnelle et classements sociaux : un ou deux savoirs ? », in La sociologie du travail et la codification du social. Actes des IV Journées de sociologie du travail, Toulouse, PIRTTEM-CNRS, 1990.

DeSROSIÈRES A. \& THÉVENOT L. - Les catégories socioprofessionnelles, Paris, La Découverte, 1988.

DUBET F. - La galère : jeunes en survie, Paris, Fayard, 1987.

ESTABLET R. - « E.T.I. [enfant de travailleur immigré] et interculturel », in Prospective culturelle, Actes du colloque européen (Marseille, mars 1987), Bruxelles, Institut de recherche et de formation sur l'éducation et la communication (IRFEC)/Nice, Institut d'études interethniques et interculturelles (IDERIC), 1988.

GARFInKEL H. - Studies in ethnomethodology, Englewood Cliffs, NJ, Prentice-Hall, 1967.

KEIL F. C. - Concepts, kinds and cognitive development, Cambridge, MA, MIT Press, 1989.

LAGRÉE J.-C. \& LEW-FAI P. - La galère. Marginalisations juvéniles et collectivités locales, Paris, Éd. du CNRS, 1985.

LAPEYRONNIE D. - « Assimilation, mobilisation et action collective chez les jeunes de la seconde génération de l'immigration maghrébine ", Revue française de Sociologie, 28, p. 287-318.

MiQuel A. - L'islam et sa civilisation, Paris, Armand Colin.

ORIOL M. - Identités culturelles, identités nationales. Théorie et étude de cas, Paris, Université Paris-V, thèse de doctorat d'État, 1989.

STREIFF-FÉNART J. - « Les "Beurs" font leur chemin », in M.-C. de La Roncière, ed., Jeunes d'aujourd'hui. Regards sur les 13-25 ans en France, Paris, La Documentation française, 1987, p. 127-138 (« Notes et études documentaires », 4843).

TURIEL D. - The development of social knowledge. Morality and convention, Londres-New York, Cambridge University Press.

ZirotTi J.-P. - « Les immigrés et l'école. Analyse et prospective », in Prospective culturelle, Actes du colloque européen (Marseille, mars 1987), Bruxelles, Institut de recherche et de formation sur l'éducation et la communication (IRFEC)/Nice, Institut d'études interethniques et interculturelles (IDERIC), p. 193-219. - « L'affaire du foulard islamique : une entreprise de fourniture en bonnes raisons ou comment masquer l'inacceptable ", in Différences culturelles et laïcité, Actes de la table ronde de l'Association pour la recherche interculturelle (ARIC), Paris.

ZirotTI J.-P. \& Novi M. - La scolarisation des enfants de travailleurs immigrés. Évaluation, sélection et orientation, Nice, Université de Nice, Institut d'études interethniques et interculturelles, Rapport de recherche, multigr.

\section{NOTES}

1. Martial Bild, dirigeant du Front national de la jeunesse, dénonçait dans le journal Le Monde: « les responsables de la désagrégation de l'enseignement et de l'explosion de l'insécurité dans les établissements scolaires: militants des formations socialo-trotskistes, "red-skins" du Parti 
communiste, gangs immigrés et bandes tribales [...] La sociologie et la physionomie des pilleurs et des casseurs ne laissent planer aucun doute sur l'absence des militants jeunes du Front national de ces débordements intolérables » (jeudi, 15/11/90, p. 13).

2. Voir sur ce point la contribution de T. Blöss \& I. Feroni qui, dans ce même ouvrage, se livrent à un examen critique des modes de catégorisation de la jeunesse.

3. «Constitués, de façon indifférenciée, en objet d'un discours social entièrement construit à partir des problèmes sociaux (scolarisation, délinquance, chômage) qu'ils posent à la nation de résidence, les enfants des immigrés en sont venus, à partir des années 1980, à se reconnaître et à se faire reconnaître comme une fraction spécifique de la jeunesse française. " (Streiff-Fénart, 1987 : p. 136.) L'indifférenciation alléguée est cependant limitée aux "jeunes Maghrébins » puisque c'est des «Beurs» qu'il est question ainsi que l'indique le titre du paragraphe dont relève cet extrait.

4. L'usage péjoratif de l'adjectif « bronzé » en représente un exemple significatif.

5. Souligné par nous. 